\title{
Pflegeroboter aus Sicht der Geriatrie
}

\author{
Christoph Gisinger
}

\section{Zusammenfassung}

Im Rahmen von zwei großen EU-Forschungsprojekten (HOBBIT und STRANDS) wurden Feldstudien über den Langzeiteinsatz von Robotersystemen in der geriatrischen Pflege- und Rehabilitationseinrichtung Haus der Barmherzigkeit und in Privatwohnungen von alleine lebenden Menschen im Alter über 75 Jahre durchgeführt. Die geriatrischen Patienten reagierten im Allgemeinen offen und aufgeschlossen, wesentlich zurückhaltender waren - vor allem anfangs - die Mitarbeiterinnen und Mitarbeiter. Die technischen Projektpartner bewerteten den Langzeiteinsatz unter Alltagsbedingungen äußerst positiv, weil die Assistenzrobotersysteme außerhalb des Labors unter Real-Life-Bedingungen überhaupt langfristig und weitgehend autonom funktionsfähig waren. Die Nutzer (Pflegepatienten, deren Angehörige und Mitarbeiter) reagierten eher enttäuscht, weil die eingesetzten Prototypen nicht ausreichend robust, rasch und verlässlich funktionierten und dadurch zu wenig Unterstützung im Alltag und praktischer Nutzen erlebbar war. Im weiteren Projektverlauf konnten Ängste der Mitarbeiter entkräftet werden, dass Pflegepersonen durch Roboter in absehbarer Zeit ersetzt werden könnten. Für Teilaufgaben oder unterstützend bzw. im Tandem wird jedoch ein großes Potenzial für intelligente technische Lösungen begrüßt. Seitens der Nutzer bzw. Pflegepatienten zeigte sich keine grundsätzliche Ablehnung von robust und sicher funktionierenden Robotern und sogar eine Präferenz für technische Hilfe

\section{Gisinger $(\square)$}

Department für Gesundheitswissenschaften und Biomedizin, Zentrum für Geriatrische Medizin und Pflege, Donau-Universität Krems, Krems, Österreich

E-Mail: christoph.gisinger@donau-uni.ac.at

C. Gisinger

Akademie für Altersforschung am Haus der Barmherzigkeit, Wien, Österreich

(C) Der/die Autor(en) 2018

O. Bendel (Hrsg.), Pflegeroboter, https://doi.org/10.1007/978-3-658-22698-5_6 
statt Personenhilfe, wenn es um den Intimbereich (z. B. Körperpflege, Ausscheidung) geht und dadurch Autonomie und Selbstständigkeit erhalten werden können. Hier gibt es allerdings technische, wirtschaftliche, Sicherheits- und ethische Probleme, die noch gelöst werden müssen. „Pflegeroboter“ werden kommen, auch wenn noch ein langer Weg vor uns liegt und die genaue Funktionalität noch einer Klärung bedarf.

\subsection{Einleitung}

Sowohl der Begriff „Pflege“ als auch der Begriff „Roboter“ wird unterschiedlich verwendet und verstanden. Unter der Bezeichnung „Pflege“ wird sowohl der gesamte Dienstleistungsbereich der mobilen oder (teil-)stationären Betreuung im Gesundheitsbzw. Sozialbereich verstanden als auch bestimmte in vielen Ländern gesetzlich stark geregelte Berufsbilder und andererseits auch Tätigkeiten, die von nicht ausgebildeten Personen, also Laien, in Familien oder anderen informellen Bereichen übernommen werden. Die im Englischen gebräuchliche Unterscheidung von „Care“ und „Nursing“ (Nurses ICo) wird ins Deutsche als „Betreuung“ und „Pflege“ übersetzt, allerdings ist diese Differenzierung in der Alltagssprache nicht wirklich angekommen.

Ähnlich beliebig verhält es sich mit dem Begriff „Roboter“, der ein weites Spektrum umfasst; von programmierbaren deterministischen Maschinen (klassische „Industrieroboter") über z. B. in der Medizin eingesetzte Manipulatoren (Operationsroboter), die von Chirurgen gesteuert werden, bis zu mit komplexen Sensoren bestückten stationären oder mobilen Systemen, die Signale erkennen, differenziert verarbeiten und darauf reagieren können und deren Aussehen oft auch anthropomorphe Merkmale aufweist.

Die Bezeichnung „Pflegeroboter“ ist daher häufig eine schwammige Projektionsfläche von Wünschen und Ängsten. In diesem Beitrag werden aus Sicht der Geriatrie und Medizin Erwartungen und Erfahrungen mit komplexen technischen Systemen in einer geriatrischen Langzeitpflege- und Rehabilitationseinrichtung zusammengefasst, die Menschen mit erhöhtem Pflegebedarfsrisiko oder tatsächlichem Pflegebedarf unterstützen und zu Hause oder in Institutionen eingesetzt werden können.

\subsection{Wo können Technik und Robotik im Bereich Geriatrie und Pflege nützlich sein?}

Im Jahr 2008 untersuchten wir in einem vierwöchigen Projekt gemeinsam mit einem Team von Mechatronikern des Austrian Research Center mittels Workshops und strukturierter teilnehmender Beobachtung unter Einbindung verschiedener Berufsgruppen des Pflege- und Rehabilitationsbereichs des Hauses der Barmherzigkeit, für welche Aufgaben technische Systeme oder Roboter eine Hilfe sein könnten (Helnwein et al. 2008). Neben sehr spezifischen Anforderungen, wie z. B. bei Patienten mit Kontrakturen, wurden für Menschen mit Pflegebedarf zusammenfassend folgende Bereiche als vielversprechende weitere Stoßrichtung identifiziert: Sturzprophylaxe und Sturzerkennung, Unterstützung 
der Kommunikation, Vermittlung von Erlebnissen und Unterhaltung, Unterstützung bei (kognitiver) Therapie/Training, Hol- und Bringdienste, Bewohner-/Patiententransport in der Einrichtung, automatisierte Unterstützung bei der Pflegedokumentation, Unterstützung der professionellen Pflege bei Hebe- und Kraftaufgaben, automatisierte Medikamentenverabreichung mit Dokumentation des Durchführungsnachweises, apportierender Visitenverbandswagen mit „Dritte-Hand“-Funktion. Natürlich stellt sich die technische, finanzielle, ethische, rechtliche und organisatorische Realisierbarkeit sehr unterschiedlich dar. In den Folgejahren ergab sich die Gelegenheit, in einigen dieser Bereiche gemeinsam mit Partnern aus Universitäten und Industrie Projekte mit dem Ziel zu verwirklichen, Lösungsansätze für die Probleme Sturz bzw. Sicherheit zu entwickeln oder Möglichkeiten bei der Unterstützung von rehabilitativen Therapien aufzuzeigen. Dazu kamen auch Informations-, Kommunikations- und Unterhaltungsfunktionen.

\subsection{Sturz: Auslöser von Pflegebedürftigkeit}

Stürze gehören zu den wichtigen geriatrischen Syndromen und sind für rund die Hälfte aller Traumata im Alter (Sterling et al. 2001) mit entsprechenden Folgen für Pflegebedürftigkeit und Mortalität (Alexander et al. 1992) verantwortlich. Laut dem Österreichischen Seniorenbericht (Winkler und Wirl 2012) stürzen rund die Hälfte der über 80-Jährigen mindestens einmal im Jahr, von denen 10 bis $20 \%$ auch Verletzungen erleiden, bei etwa $5 \%$ kommt es sogar zu Knochenbrüchen. Eine Befragung im Rahmen der Österreichischen Interdisziplinären Hochaltrigen-Studie (Stückler und Ruppe 2015) gibt eine Sturzhäufigkeit bei über 80-Jährigen innerhalb der letzten drei Monate von lediglich 15,6\% an, von denen allerdings 47,7 \% zumindest leicht (Hautabschürfungen, blaue Flecken etc.) und 15,4\% schwer (Knochenbrüche und ähnlicher Schweregrad) verletzt worden sind.

Die klinische Erfahrung von Unfallchirurgen zeigt, dass Stürze von alleine lebenden SeniorInnen mit schweren Sturzfolgen oft erst nach erheblicher Latenzzeit vom Umfeld bemerkt werden und daher Hilfeleistung oft erst stark verzögert erfolgt. Zahlreiche Studien untersuchten den Einsatz verschiedener technischer Systeme zur Sturzerkennung, wie beispielsweise Vibrationssensoren (Zigel et al. 2009) oder Messung der Veränderung des elektrischen Feldes (Rimminen et al. 2010), verschiedene am Körper (Armband, Gürtelschnalle etc.) getragene Sensoren (Bianchi et al. 2009) und verschiedene Konzepte mit Einsatz der Mobil- bzw. Smartphone-Technologie (Williams et al. 2013; Poku 2015). Allerdings haben sich diese Systeme in der Praxis bisher unter den Erwartungen etabliert, zum Teil wegen des Installationsaufwands, oder - vor allem bei am Körper getragenen Sensoren - wegen mangelnder Akzeptanz durch die Betroffenen, vor allem in Situationen (Bad, nächtlicher WC-Gang), bei denen das Sturzrisiko erhöht ist. Ein weiteres Problem sind die häufigen Fehlalarme, was nicht zur Akzeptanzsteigerung der Betroffenen, ihrer Familien oder der Betreuungs- oder Notfalldienste beiträgt. Ein neuer Ansatz sollte daher hohe Spezifität (=wenige Fehlalarme) und hohe Sensitivität (=keine unerkannten Stürze) aufweisen. 


\subsection{Die Entwicklung des Assistenzroboters HOBBIT}

Gemeinsam mit sechs Partnern starteten wir 2011 das Projekt HOBBIT (FP7/2007-2013, grant agreement $n^{\circ}$ 288.146). Das mobile, autonom navigierende Assistenzsystem hatte das Ziel, Stürze von zu Hause alleine lebenden SeniorInnen zu erkennen, Fehlalarmierungen durch die Möglichkeit von Rückfragedialogen und Lageanalyse aus mehreren Perspektiven zu minimieren und verschiedene nützliche Funktionen für den Alltag zur Verfügung zu stellen, um sicherzustellen, dass Akzeptanz und langfristiger Einsatz des Systems unterstützt werden. Wichtig war, dass die potenziellen Nutzer von Anfang an bei Konzeption und Designentwicklung eingebunden wurden (Koertner et al. 2014), woraus zunächst der Bau eines ersten Prototyps (siehe Abb. 6.1) und darauf aufbauend eines zweiten Prototyps erfolgte, welcher dann tatsächlich unter Realbedingungen in den Privathaushalten von SeniorInnen über mehrere Wochen eingesetzt werden konnte (siehe Abb. 6.2).

HOBBIT Prototyp 2 verfügte über eine zum Boden parallele Tiefenkamera, einen Bildschirm mit einer RGB-D-Kamera (ASUS Xtion auf $120 \mathrm{~cm}$ Höhe montiert) als „Kopf“, einen Touchscreen auf etwa halber Höhe und einen Greifarm (siehe Abb. 6.3), weitere technische Details siehe (Fischinger et al. 2016). Die Gesamthöhe betrug $125 \mathrm{~cm}$, die Gesamtbreite einschließlich Greifarm in Ruheposition $56 \mathrm{~cm}$. Die Nutzerinteraktion erfolgte über ein multimodales User Interface bestehend aus dem grafischen
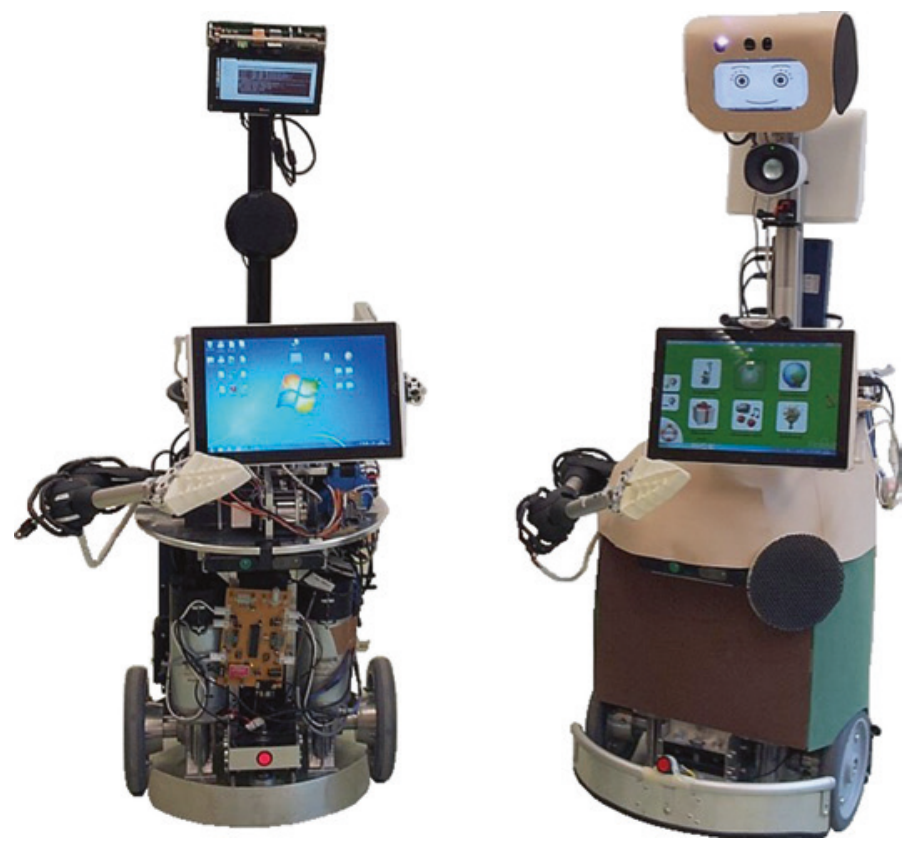

Abb. 6.1 HOBBIT Prototyp 1 - Varianten 
Abb. 6.2 HOBBIT Prototyp 2 in einer Privatwohnung

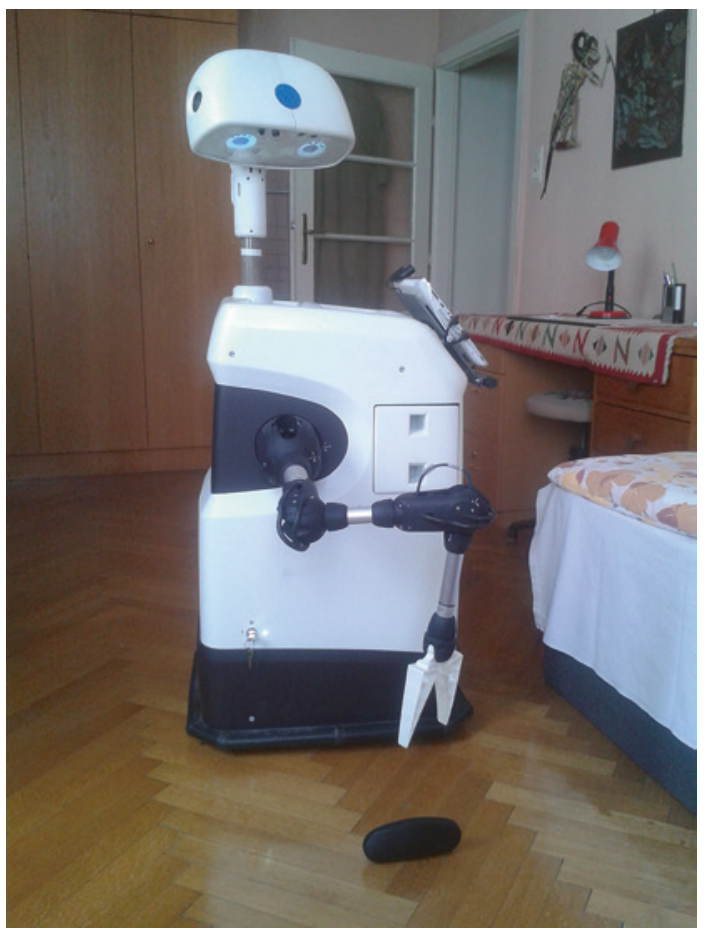

Touchscreen, automatischer Spracherkennung sowie Text-zu-Sprache- und GestenErkennungsinterface. Als Funktionen wurden Unterhaltungs- und Erlebniselemente (Musik, Radio, Audiobücher, Spiele, vorinstallierte Internetdienste, Fitnessfunktionen) angeboten, verschiedene Erinnerungsfunktionen (z. B. Medikamenteneinnahme), Kommunikationsmöglichkeiten (Videotelefonie, Zugang zu Ambient-Assisted-Living-[AAL]Umgebungen, Ruftasten) und Notfallfunktionen einschließlich automatischer Sturzerkennung auf Basis von Bildanalyse aus mehreren Perspektiven und bei Notfall- oder Sturzverdacht Dialogfunktionen mit Eskalationsalgorithmen zur allfälligen Verifizierung des Ereignisses, bevor ein externer Alarm abgesetzt wird. Außerdem verfügte HOBBIT über verschiedene Prozeduren im Sinne der Sturzprävention (kleinere Gegenstände vom Boden aufheben und/oder transportieren, Objekte suchen, wie vom Nutzer programmiert), siehe dazu auch Videoclip (v4racintuvienna vrt.).

Unsere in Wiener Privatwohnungen durchgeführte Feldstudie umfasste sieben alleine lebende Nutzer (sechs Frauen, ein Mann) mit einem Durchschnittsalter von 79 Jahren (75 bis 88 Jahre). Die Einsatzzeit pro Nutzer umfasste mindestens 3 Wochen, womit wir über Erfahrungen des Langzeiteinsatzes von HOBBIT in Privathaushalten über rund 5 Monate verfügen. Fünf der Teilnehmer hatten in den vorangegangenen zwei Jahren mäßige bis schwerere Mobilitätsprobleme, zwei hatten bereits davor Stürze zu Hause, bei denen Fremdhilfe notwendig geworden war (Pripfl 2016). Die Evaluation erfolgte 
Abb. 6.3 STRANDS

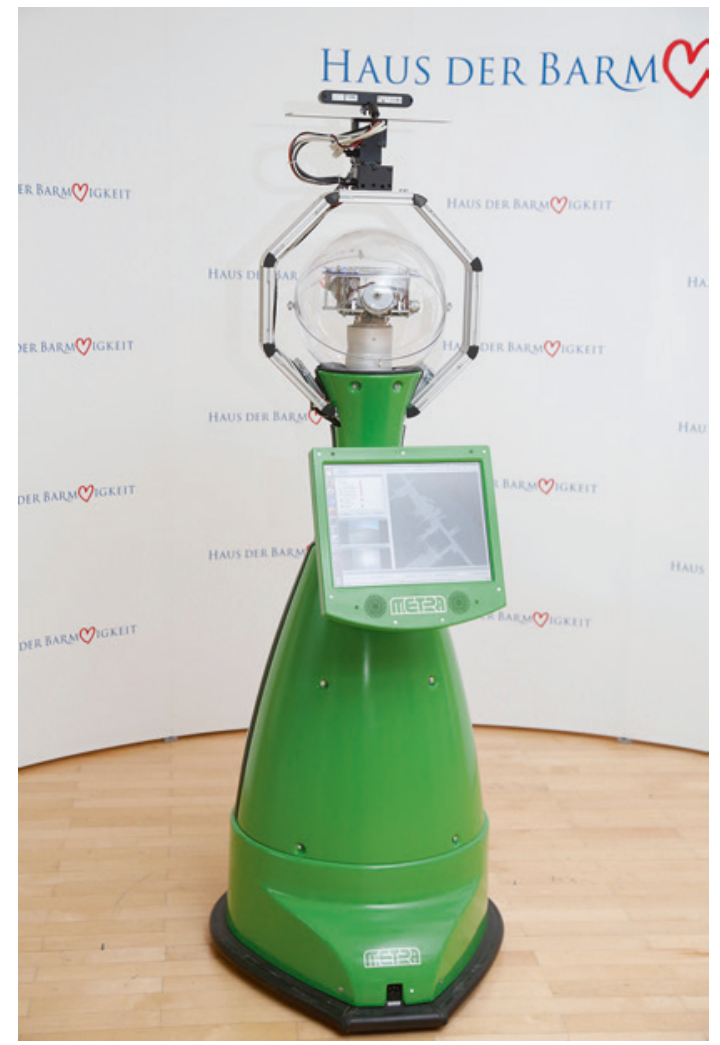

mittels qualitativer Interviews vor, unmittelbar nach dem Einsatz von Hobbit sowie einige Wochen später. Die Funktionen, Gegenstände vom Boden aufheben und transportieren, Notfälle erkennen und Erinnerungsmeldungen einstellen zu können, wurden positiv bewertet. Ebenfalls positiv war die Beurteilung von Usability und intuitiver Handhabung. Negative Rückmeldungen bezogen sich auf die mangelnde Robustheit des Prototyps mit häufigen Fehlfunktionen, Probleme mit der automatischen Notfallerkennung (zu viele falsch positive Ereignisse) und die generelle Langsamkeit des Systems (niedrige Fahrgeschwindigkeit und langsamer Greifarm), was sowohl mit Sicherheitsbedenken als auch mit mechanischen, technischen und finanziellen Limitierungen bei der Prototypentwicklung zu tun hatte. Ebenfalls kritisch bewertet wurde die Unverlässlichkeit der Sprach- und Gesteneingabe, weswegen überwiegend der Touchscreen zur Verwendung kam. Die generelle Einstellung zu Robotern wurde mittels der Negative Attitudes Towards Robots Scale (NARS) (Nomura et al. 2006) ermittelt und zeigte nur in einer von mehreren Subkategorien (,negative attitude toward emotions in interaction with robots") einen grenzwertig signifikanten Anstieg, während sich das Sicherheitsgefühl mittels einer gekürzten Version der Falls Efficacy Scale (FES) (Yardley et al. 2005) nicht verändert hat. 
Zusammenfassend zeigte sich die grundsätzliche Akzeptanz, Praktikabilität und Bedienbarkeit eines solchen Assistenzroboters im Langzeiteinsatz in Privatwohnungen von älteren Personen. Allerdings gab es bedeutende technische Limitationen, nicht zuletzt durch das Ziel, ein leistbares System zu entwickeln. Diese technischen Grenzen erscheinen jedoch bei entsprechendem Mitteleinsatz für die Entwicklung eines weiteren Prototyps grundsätzlich lösbar. Da viele der mechanischen und Sicherheitskomponenten speziell weiterentwickelt werden müssen, ist die Leistbarkeit eines solchen Systems für durchschnittliche Privathaushalte in entwickelten Industrieländern erst bei einer Großserienproduktion zu erwarten. Die Überwindung dieses „Henne-Ei-Problems“ (also hoher Preis wegen geringer Nachfrage und geringe Nachfrage wegen hohem Preis) stellt die eigentliche Herausforderung dar und erfordert eine bedeutende mehrjährige, nicht risikolose Anfangsinvestition und einen ,langen Atem“, die nur durch finanziell sehr potente Technologiefirmen, große im Bereich Pflege und Gesundheit tätige Dienstleister oder durch Förderungen von Forschungs- und Entwicklungsprogrammen aus öffentlichen Mitteln aufgebracht werden können.

\subsection{STRANDS zur Unterstützung der Physiotherapie}

Im Jahr 2013 starteten wir mit sieben weiteren Projektpartnern (FP7/2007-2013, Cognitive Systems and Robotics, grant agreement $n^{\circ}$ 600623) das über vier Jahre laufende Projekt STRANDS (Spatio-Temporal Representation and Activities for Cognitive Control in Long-Term Scenarios), welches Software für eine SCITOS G5 (Metrolabs Robotics, Ilmenau, D), eine mobile Roboterplattform (siehe Abb. 6.3), entwickelte, die mit verschiedenen spezialisierten Sensoren ausgestattet wurde, um Menschen sehen, erkennen, diesen und anderen Hindernissen ausweichen, die Umgebung topographisch erfassen und autonom in öffentlichen und halböffentlichen Innenräumen navigieren zu können.

Im Rahmen von Feldstudien erfolgten Langzeiteinsätze in einem Bürogebäude und im Foyer, in Warte- und Gangbereichen der Wiener Pflegeeinrichtung Haus der Barmherzigkeit über mehrere Monate. Neben Auskunfts-, Wegweiser- und Unterhaltungsfunktion erfolgte auch ein Einsatz zur Unterstützung der Ergo- und Physiotherapie (Gerling et al. 2016), die neben kognitivem Training im Speziellen in der Begleitung von Demenz-Nordic-Walking-Gruppen bestand (Hebesberger et al. 2016b), siehe auch Videoclip (gisinger).

Die Langzeit-Stationierung eines autonom navigierenden Roboters im gesamten Erdgeschoss der Pflege- bzw. Rehabilitationseinrichtung mit 350 Betten, 465 Mitarbeitern, Ambulanzen und sonstigen Einrichtungen (z. B. Betriebskindergarten, Kaffeehaus, Seminarräume, Friseur) mit entsprechendem sehr geschäftigem Fußgänger- und Rollstuhlfahrerverkehr stellte eine große Herausforderung dar. Entsprechende Ansprüche ergaben sich daher für die Entwicklung der Navigationssoftware, was durch die Modellierung von dynamischen Menschenmengen (Trautman 2013) und durch spezielle Zeitalgorithmen bei der Navigationsberechnung (Kollmitz 2015) erreicht werden konnte, 
aber auch dadurch, dass in bestimmten Situationen Menschen um Hilfe ersucht werden (Kruse 2010), beispielsweise durch die Bitte, einige Zentimeter weiter geschoben zu werden. Im STRANDS-Projekt wurden diese Navigationsherausforderungen auch durch die kontinuierliche Selbst-Adaptierung der Navigation und automatisiertes Lernen aus vorherigen Situationen (Dondrup et al. 2015) bewältigt. Eine völlig neue Schlüsselherausforderung stellte das Erkennen von Menschen in unmittelbarer Nähe dar, insbesondere, wenn es sich um Rollstuhlfahrer handelte. Da die Programmierung der Sicherheit in dieser Umgebung von Menschen mit hoher Vulnerabilität besonders priorisiert worden ist, kam es nicht selten zum Übergang in den Wartemodus bzw. zu einer „stotternden“ Funktionalität. Unsere Beobachtungen des sich gegenseitig beeinflussenden Roboter-Mensch-Bewegungsmusters erinnerte an das ,tänzerische“ Ausweichen in Menschenmengen zur Kollisionsvermeidung, wie zum Beispiel in Fußgängerzonen (Gerling et al. 2016).

Im STRANDS-Projekt wurde der Roboter auch zur Unterstützung der Ergo- und Physiotherapie von Menschen mit Demenz eingesetzt. Generell zeigten die TherapeutInnen eine positive Einstellung zum Einsatz eines Roboters, insbesondere hinsichtlich verschiedener Unterhaltungsfunktionen, wie zum Beispiel der Animation von Menschen mit fortgeschrittener Demenz, mitzusingen, mitzuklatschen und mitzutanzen (Hebesberger et al. 2016a). Rund $60 \%$ der DemenzpatientInnen versuchten, aktiv mit dem Roboter zu interagieren, wobei sie meist dazu seitens der TherapeutInnen Unterstützung benötigten (z. B. Bedienung des Touchscreens), obwohl das grafische Interface bewusst sehr simpel gestaltet worden ist. Insbesondere zeigte sich, dass ältere Individuen auf Icons drückten, ohne sie loszulassen, was die entsprechende Funktion nicht aktivieren konnte. Die bei TherapeutInnen und Betroffenen beliebteste Funktion war die Demenz-Nordic-WalkingGruppe. Betroffene mit fortgeschrittener Demenz erhalten in unserer spezialisierten Einrichtung verschiedene therapeutische Interventionen, darunter die Nordic-WalkingGruppe (innerhalb des Gebäudes durch die weitläufigen Foyers und Gänge) mit dem Ziel, die Mobilität zu erhalten, Reize, Erlebnisse und Ablenkung anzubieten und ihnen Interaktionen mit anderen zu ermöglichen. Im STRANDS-Projekt wurde der Roboter als Schrittmacher eingesetzt, er fuhr der Gruppe voraus, spielte populäre Wanderlieder, die zum Mitsingen animierten, rhythmisierte das Gangbild und sorgte für Aufmerksamkeit und Unterhaltung.

\subsection{Diskussion}

Die Frage ist nicht, ob, sondern wie genau und wann ,intelligente“, ,,autonome“ technische Systeme bei der Unterstützung von Menschen mit erhöhtem Risiko von oder bereits tatsächlich eingetretener Pflegebedürftigkeit eine wichtige Rolle spielen werden (Gisinger et al. 2016). Die Vorstellung, dass ein dann als „Pflegeroboter“ bezeichnetes System genau die Tätigkeit einer Pflegeperson abbildet, erscheint jedoch genauso naiv wie die Vorstellung, dass im Haushalt statt einer Geschirrspülmaschine ein anthropomorpher Roboter mit zwei Händen in einem Spülbecken schmutziges Geschirr abwäscht. 
Pflegeroboter, die physische und Kraft erfordernde Tätigkeiten übernehmen, entsprechen zwar den Wunschvorstellungen vieler Pflegepersonen, erscheinen aber am schwierigsten realisierbar, da bei einem Durchschnittsgewicht von zumindest $75 \mathrm{~kg}$ einer pflegebedürftigen Person unter Berücksichtigung der Hebelwirkung und diverser Sicherheitsreserven das entsprechende Robotersystem ein noch höheres Gewicht (oder eine fixe Verbindung mit dem Gebäude) haben müsste und wahrscheinlich ziemlich unförmig wäre, was sich mit der Enge von Wohn- und Innenräumen oft nicht vereinbaren lässt. Inwieweit sich die Unterstützung von Pflegepersonen mittels Exoskeletten bei den angesprochenen Aufgaben durchsetzen wird, erscheint angesichts der bisherigen Erfahrungen mit vergleichsweise geringen Lasten (Huysamen et al. 2018) ebenso fraglich wie deren Bezeichnung als „Pflegeroboter“.

Daher erscheinen „Pflegeroboter" mit Aufgaben in den Bereichen Sicherheit, Therapie, Holen und Bringen, Vermittlung von Erlebnissen, Information, Kommunikation und Unterhaltung in absehbarer Zeit eher realisierbar. Die Robbe Paro stellt einen Ansatz dar, wie Roboter in der Therapie von Menschen mit Demenz eingesetzt werden können (Joranson et al. 2016). Ähnlich wie im STRANDS-Projekt zeigen die Erfahrungen mit Paro deren Bedeutung im Rahmen von Gruppentherapien (Joranson et al. 2016) mit entsprechenden Auswirkungen auf die Gruppendynamik. Neben speziellen Therapieaufgaben und der Funktion als Animator ist wohl der Informations-, Kommunikations- und Unterhaltungsbereich am vielversprechendsten. Hohes Potenzial hat auch die Weiterentwicklung der Sensorik für Sicherheitsaufgaben, wie zum Beispiel zur Notfall- und Sturzerkennung. Wir konnten zeigen, dass die Akzeptanz in diesem Bereich sehr hoch ist, wenn die Systeme auch wirklich verlässlich funktionieren. Die Projekte HOBBIT und STRANDS sind zwei der sehr seltenen Feldstudien, in welchen Roboter unter realen Alltagsbedingungen über einen langen Zeitraum (mehrere Wochen) ohne technische Betreuung tatsächlich eingesetzt worden sind: HOBBIT in Privatwohnungen von alleine lebenden SeniorInnen und STRANDS im Foyer- und Gangbereich einer großen geriatrischen Pflege- und Rehabilitationseinrichtung. Während die Projektpartner aus dem Bereich Technik und Computerwissenschaft ihre Erwartungen bezüglich des Einsatzes ihrer Robotersysteme außerhalb des Labors mit großer Zufriedenheit verfolgten, machte sich bei den aus der Praxis des Geriatrie- und Pflegebereiches stammenden Projektpartnern und bei den Nutzern Ernüchterung bis Enttäuschung breit, weil viele der Funktionalitäten als viel zu plump oder zu langsam empfunden wurden oder die Erwartungshaltung an die Vielfalt der eingesetzten Sensoren und deren Integration zu hoch war. Dies hing allerdings mit dem begrenzten Budget für die Prototypentwicklung im HOBBIT-Projekt und mit der Verwendung einer zugekauften Roboterforschungsplattform aus Serienproduktion im STRANDS-Projekt mit entsprechenden Limitationen zusammen.

Natürlich ergeben sich auch wichtige ethische Fragestellungen, die in diesem Buch an anderer Stelle ausführlicher zu Wort kommen und in unseren Projekten ebenfalls eingehend reflektiert worden sind (Körtner 2016). Angesichts der auf uns zukommenden Herausforderung, wenn die „Babyboomer“ nach Erreichen ihres Pensionsalters bald zu 
„Gerontoboomern“ geworden sein werden, und angesichts des zu erwartenden Mangels an Fachpersonal im Gesundheits- und Pflegebereich (Famira-Müelberger et al. 2017) stellt sich die Frage, ob künstliche Intelligenz und Expertensysteme eine Rolle bei der Unterstützung und Dokumentation der Betreuungs- und Pflegetätigkeiten durch nicht ausgebildete Menschen spielen könnten, vielleicht in Analogie zu Konzepten, wie sie in automatischen Laien-Defibrillatoren verwendet werden (Hosmans et al. 2008). Dieser Beitrag und dieses Buch versuchen, Anregungen für die zukünftige Forschung und Entwicklung von Pflegerobotern zu geben. Wir können gespannt sein, welche Konfiguration, welcher Funktionsumfang, welches Aussehen und welche Einsatzgebiete sich letztendlich durchsetzen werden.

Danksagung Herzlicher Dank an alle Partner im HOBBIT- und STRANDS-Projekt. Besondere Erwähnung verdient das Team der Akademie für Altersforschung (AAF) am Haus der Barmherzigkeit, das das Projekt durchgeführt, begleitet und wissenschaftlich aufgearbeitet hat: Daliah Batko-Klein, Matei Capatu, Denise Hebesberger, Tobias Körtner, Victoria MühleggerStaus, Jürgen Pripfl, Veronika Schauer, Alexandra Schmid, und das interdisziplinäre Team der geriatrischen Pflege- und Rehabilitationseinrichtung Haus der Barmherzigkeit in Wien

\section{Literatur}

Alexander, B. H., Rivara, F. P., \& Wolf, M. E. (1992). The cost and frequency of hospitalization for fall-related injuries in older adults. American Journal of Public Health, 82(7), 1020-1023.

Bianchi, F., Redmond, S. J., Narayanan, M. R., Cerutti, S., Celler, B. G., \& Lovell, N. H. (2009). Falls event detection using triaxial accelerometry and barometric pressure measurement. Conference proceedings of the IEEE engineering in medicine and biology society, 2009, 6111-6114.

Dondrup, C., Belotto, N., Hanheide, M., Eder, K., \& Leonhards, U. (2015). A computational model of human-robot spatial interactions based on a qualitative trajectory calculus. Robotics, 4(1), 63-102.

Famira-Müelberger, U., Firgo, M., Fritz, O., \& Streicher, G. (2017). Österreichisches Institut für Wirtschaftsforschung - Austrian Institute of Economic Research.

Fischinger, D., Einramhof, P., Papoutsakis, K., Wohlkinger, W., Mayer, P., Panek, P., et al. (2016). Hobbit, a care robot supporting independent living at home: First prototype and lessons learned. Robotics and Autonomous Systems, 75(A), 60-78.

Gerling, K., Hebesberger, D., Dondrup, C., Kortner, T., \& Hanheide, M. (2016). Robot deployment in long-term care : Case study on using a mobile robot to support physiotherapy. Zeitschrift für Gerontologie und Geriatrie, 49(4), 288-297.

Gisinger, C., \& Pripfl, J. (2016). Alter schützt vor Technik nicht [Age is no protection against technology?!]. Zeitschrift fur Gerontologie und Geriatrie, 49(4), 271-273.

gisinger c. AAF STRANDSInfovideo Y3 EN. https://www.youtube.com/watch?v=8xhr8woFtfE. Zugegriffen: 07. Febr. 2018.

Hebesberger, D., Dondrup, C., \& Koertner, T. (2016a). Proceedings of HRI.

Hebesberger, D., Koertner, T., Gisinger, C., Pripfl, J., \& Dondrup, C. (2016b). 11th ACM/IEEE International conference on Human-Robot Interaction (HRI). 
Helnwein, B., Gisinger, C., Mina, S., Fugger, E., Koelbl-Catic, E., \& Lang, A. (2008). Akademie für Altersforschung am Haus der Barmherzigkeit und Austrian Research Center: Bundesministerium für Verkehr, Innovation und Technologie, benefit- Programm.

Hosmans, T. P., Maquoi, I., Vogels, C., Courtois, A. C., Micheels, J., Lamy, M., et al. (2008). Safety of fully automatic external defibrillation by untrained lay rescuers in the presence of a bystander. Resuscitation, 77(2), 216-219.

Huysamen, K., Looze, M. de, Bosch, T., Ortiz, J., Toxiri, S., \& O’Sullivan, L. W. (2018). Assessment of an active industrial exoskeleton to aid dynamic lifting and lowering manual handling tasks. Applied Ergonomics, 68, 125-131.

Joranson, N., Pedersen, I., Rokstad, A. M., \& Ihlebaek, C. (2016a). Change in quality of life in older people with dementia participating in Paro-activity: A cluster-randomized controlled trial. Journal of Advanced Nursing, 72(12), 3020-3033.

Joranson, N., Pedersen, I., Rokstad, A. M., Aamodt, G., Olsen, C., \& Ihlebaek, C. (2016b). Group activity with Paro in nursing homes: Systematic investigation of behaviors in participants. International Psychogeriatric, 28(8), 1345-1354.

Körtner, T. (2016). Ethical challenges in the use of social service robots for elderly people. Zeitschrift für Gerontologie und Geriatrie, 49(4), 303-307.

Koertner, T., Schmid, A., Batko-Klein, D., Gisinger, C., \& Vincze, M. (2014). Meeting requirements of older users? Robot prototype trials in a home-like environment. Universal access in human-computer interaction. Aging and assistive environments. Lecture Notes Computer Science, 2014(8515), 660-671.

Kollmitz, M. (2015). European Conference on Mobile Robots (ECMR) (S. 1-6).

Kruse, T. (2010). IEEE International Workshop on Robot and Human Interactive Communication (S. 192-197).

Nomura, T., Kanda, T., \& Suzuki, T. (2006). Experimental investigation into influence of negative attitudes toward robots on human-robot interaction. AI \& Society, 20, 138-150.

Nurses ICo. Definition of nursing. http://www.icn.ch/who-we-are/icn-definition-of-nursing/. Zugegriffen: 04. Febr. 2018.

Poku, M. (2015). The promise of smartphone fall detection solutions for falls in older adults. Journal of the American Geriatrics Society, 63(9), 1969-1970.

Pripfl, J., Kortner, T., Batko-Klein, D., Hebesberger, D., Weninger, M., \& Gisinger, C. (2016). Social service robots to support independent living: Experiences from a field trial. Zeitschrift für Gerontologie und Geriatrie, 49(4), 282-287.

Rimminen, H., Lindstrom, J., Linnavuo, M., \& Sepponen, R. (2010). Detection of falls among the elderly by a floor sensor using the electric near field. The IEEE Transactions on Information Technology in Biomedicine, 14(6), 1475-1476.

Sterling, D. A., O'Connor, J. A., \& Bonadies, J. (2001). Geriatric falls: Injury severity is high and disproportionate to mechanism. The Journal of Trauma, 50(1), 116-119.

Stückler, A., \& Ruppe, G. (2015). Österreichische Plattform für Interdisziplinäre Alternsfragen. Wien.

Trautman, P. (2013). IEEE International conference on robotics and automation (S. 2153-2160).

v4racintuvienna vrt. Hobbit robot as future help at home. https://www.youtube.com/ watch?v=KAvkv6ygVFM. Zugegriffen: 07. Febr. 2018.

Williams, V., Victor, C. R., \& McCrindle, R. (2013). It is always on your mind: Experiences and perceptions of falling of older people and their carers and the potential of a mobile falls detection device. Current Gerontology and Geriatrics Research, 2013, 295073. 
Winkler, E. P. P., \& Wirl, C. (2012). Bundesministerium für Gesundheit (S. 19).

Yardley, L., Beyer, N., Hauer, K., Kempen, G., Piot-Ziegler, C., \& Todd, C. (2005). Development and initial validation of the Falls Efficacy Scale-International (FES-I). Age and Ageing, 34(6), 614-619.

Zigel, Y., Litvak, D., \& Gannot, I. (2009). A method for automatic fall detection of elderly people using floor vibrations and sound-proof of concept on human mimicking doll falls. IEEE Transactions on Biomedical Engineering, 56(12), 2858-2867.

Christoph Gisinger ist Professor für Geriatrie und Leiter des gleichnamigen Zentrums an der Universität für Weiterbildung Donau-Universität Krems und Gesamtgeschäftsführer der gemeinnützigen Haus der Barmherzigkeit-Gruppe, die im Großraum Wien/Niederösterreich an 24 Standorten Behinderteneinrichtungen, Pflegeheime, Pflegekrankenhäuser und Geriatrische Rehabilitationseinrichtungen betreibt. Der geborene Wiener absolvierte sein Medizinstudium und seine klinische und wissenschaftliche Ausbildung zum Facharzt für Innere Medizin, Endokrinologie, Rheumatologie und Geriatrie an der Universität Wien, dem Royal Free Hospital in London und an der Medical University of South Carolina in Charleston, SC, sowie seine Management-Ausbildung an der Wirtschaftsuniversität Wien, dem Managementzentrum St. Gallen und der Columbia University New York.

Open Access Dieses Kapitel wird unter der Creative Commons Namensnennung 4.0 International Lizenz (http://creativecommons.org/licenses/by/4.0/deed.de) veröffentlicht, welche die Nutzung, Vervielfältigung, Bearbeitung, Verbreitung und Wiedergabe in jeglichem Medium und Format erlaubt, sofern Sie den/die ursprünglichen Autor(en) und die Quelle ordnungsgemäß nennen, einen Link zur Creative Commons Lizenz beifügen und angeben, ob Änderungen vorgenommen wurden.

Die in diesem Kapitel enthaltenen Bilder und sonstiges Drittmaterial unterliegen ebenfalls der genannten Creative Commons Lizenz, sofern sich aus der Abbildungslegende nichts anderes ergibt. Sofern das betreffende Material nicht unter der genannten Creative Commons Lizenz steht und die betreffende Handlung nicht nach gesetzlichen Vorschriften erlaubt ist, ist für die oben aufgeführten Weiterverwendungen des Materials die Einwilligung des jeweiligen Rechteinhabers einzuholen.

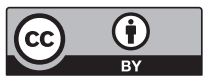

